\title{
Whole exome and targeted deep sequencing identify genome- wide allelic loss and frequent SETDB1 mutations in malignant pleural mesotheliomas
}

\author{
Hio Chung Kang ${ }^{1,2, *}$, Hong Kwan Kim ${ }^{1,3, *}$, Sharon Lee ${ }^{4}$, Pedro Mendez ${ }^{1}$, James \\ Wansoo Kim ${ }^{4}$, Gavitt Woodard ${ }^{1}$, Jun-Hee Yoon ${ }^{1}$, Kuang-Yu Jen ${ }^{5}$, Li Tai Fang ${ }^{1}$, Kirk \\ Jones $^{5}$, David M. Jablons ${ }^{1,2}$, Il-Jin Kim ${ }^{1,2}$ \\ ${ }^{1}$ Thoracic Oncology Laboratory, Department of Surgery, University of California San Francisco, San Francisco, CA, USA \\ ${ }^{2}$ Comprehensive Cancer Center, University of California San Francisco, San Francisco, CA, USA \\ ${ }^{3}$ Department of Thoracic and Cardiovascular Surgery, Samsung Medical Center, Sungkyunkwan University School of Medicine, \\ Seoul, Korea \\ ${ }^{4}$ CureSeq Inc, Brisbane, CA, USA \\ ${ }^{5}$ Department of Pathology, University of California San Francisco, San Francisco, CA, USA \\ *These authors have contributed equally to this work \\ Correspondence to: II-Jin Kim, e-mail: II-Jin.Kim@ucsf.edu \\ David M. Jablons, e-mail: David.Jablons@ucsf.edu
}

Keywords: malignant pleural mesothelioma, multiple primary cancer, genome-wide allelic loss, exome sequencing, SETDB 1

Received: October 05, 2015 Accepted: January 15, 2016 Published: January 27, 2016

\section{ABSTRACT}

Malignant pleural mesothelioma (MPM), a rare malignancy with a poor prognosis, is mainly caused by exposure to asbestos or other organic fibers, but the underlying genetic mechanism is not fully understood. Genetic alterations and causes for multiple primary cancer development including MPM are unknown. We used whole exome sequencing to identify somatic mutations in a patient with MPM and two additional primary cancers who had no evidence of venous, arterial, lymphovascular, or perineural invasion indicating dissemination of a primary lung cancer to the pleura. We found that the MPM had R282W, a key TP53 mutation, and genome-wide allelic loss or loss of heterozygosity, a distinct genomic alteration not previously described in MPM. We identified frequent inactivating SETDB1 mutations in this patient and in 68 additional MPM patients (mutation frequency: 10\%, 7/69) by targeted deep sequencing. Our observations suggest the possibility of a new genetic mechanism in the development of either MPM or multiple primary cancers. The frequent SETDB1 inactivating mutations suggest there could be new diagnostic or therapeutic options for MPM.

\section{INTRODUCTION}

Malignant pleural mesothelioma (MPM) is a rare malignancy with a highly unfavorable prognosis. Increased risk for MPM is strongly linked to exposure to asbestos or erionite [1-4]. Since asbestos had widely been used in different industries, the incidence of MPM in the United States is expected to steadily rise and peak over the next 20 years [1-4]. Clinical trials of single modality treatment with extrapleural pneumonectomy or pleurectomy, chemotherapy or radiation therapy have not significantly improved survival. Median survival ranges from 10-17 months [5-7].

The underlying genetic mechanisms of MPM development are not fully known. Molecular genetic analyses of MPMs have shown frequent deletions in chromosomes 1p, 3p, 4p, 4q, 6q, 9p, 13q, 14q, 15q, and 22q [8-10]. Certain tumor suppressor genes located in these regions have been implicated, such as $C D K N 2 A$ / $A R F$ at chromosome $9 \mathrm{p} 21, N F 2$ at $22 \mathrm{q} 12$, and $B A P 1$ at $3 \mathrm{p} 21,[4,11-14]$, but for many of these regions, the driver genes remain to be identified. Since the first report 
of transcriptome sequencing in MPM samples [15], large-scale genome analyses such as exome sequencing [16-18] and whole genome sequencing (WGS) [19-20] using MPM tissues or cultured cells identified potential molecular targets such as E2F1 [17], CUL1 [18], METrelated pathway genes, and $m S W I / S N F$ genes, the latter of which are important for histone modification and regulation [16]. These genetic alterations suggest potentially new therapeutic targets or diagnostic markers in addition to known frequent genetic alterations $(N F 2$, $C D K N 2 A$, and $B A P 1)$ in MPM.

Interestingly, many genes involved in histone modification and regulation mechanisms undergo germline or somatic mutations [16]. SETDB1, a histone methyltransferase, is reportedly a potential oncogene in lung cancer [21]. A clinical trial for the SETDB1targeting drug mithramycin is underway for both lung cancer and mesothelioma (clinicaltrials.gov). A systematic analysis of $S E T D B 1$ and other genes involved in histone modification could help identify genetic mechanisms for MPM. Furthermore, although previous large-scale genome studies suggested potential drug targets and diagnostic markers for MPM, most studies were confined to focal changes of mutations such as small substitutions or insertions and deletions. A systemic large genomic change such as genome-wide deletion has not been reported for MPM.

It is uncommon to observe multiple primary tumors along with MPM in the same patient. There are few, if any, suggested genetic mechanisms for the development of multiple cancers in a patient who has MPM. Here, we present a rare case of a patient who had multiple primary cancers including MPM and suggest a potentially novel genetic mechanism that explains this unusual development. Targeted deep sequencing identified a frequently mutated gene, $S E T D B 1$, in this patient and several others with MPM.

\section{RESULTS}

\section{Clinical characteristics of an MPM patient with two additional primary cancers}

A 62-year-old Caucasian woman presented with persistent symptoms of fever, cough, chest pain and a left lower lobe consolidation on chest radiograph despite the use of antibiotics. Six months later, computed tomography of the chest revealed a discrete mass in the left lower lobe, which fine needle aspiration showed to be non-small cell lung cancer (Figure 1A). Positron emission tomography and a bone scan revealed no evidence of distant metastasis, and the patient was referred for a thoracic surgery evaluation. Her past medical history was significant for bladder cancer treated with transurethral bladder resection eight years earlier. She was a former smoker with a 30 pack-year history and had worked for a construction company while in her forties with possible, but uncertain asbestos exposure.

Five months after her initial presentation, the patient underwent a left lower lobectomy with mediastinal lymph node dissection. On thoracotomy, scattered mass lesions were incidentally discovered on the pleural surface of the diaphragm and biopsied. Final pathology revealed a T1N0M0 lung adenocarcinoma in the left lower lobe and pleural biopsies indicated sarcomatoid pleural mesothelioma, which stained positive for calretinin (Figure 1B) and was staged as T3N0M0 because there was no evidence of venous, arterial, lymphovascular, or perineural invasion. Postoperatively, two cycles of neoadjuvant chemotherapy with carboplatin and pemetrexed resulted in some disease regression. The patient had a subsequent completion left pneumonectomy. She died from mesothelioma progression four months later.

\section{Exome sequencing of the MPM patient's tumor revealed a genome-wide allelic loss}

The development of multiple primary malignancies including MPM led us to search for underlying genetic alterations in this patient. First, we performed whole exome sequencing on the mesothelioma and adjacent normal pleural tissue as a control. Among the 11 highconfidence, non-synonymous variants identified, six were further validated by targeted deep or Sanger sequencing (Table 1). One key mutation identified was $\mathrm{R} 282 \mathrm{~W}$ in the TP53 tumor suppressor gene. R282W is a structural mutation that renders the DNA-binding domain of p53 unstable and is a common TP53 cancerrelated mutation [22-24], but has not been described in MPM. We also identified a nonsense mutation, Y249X, of SETDB1, a histone methyltransferase. SETDB1 is an oncogene frequently amplified in human lung cancers and melanomas [21, 25]. A frameshift mutation (V132fs) producing a premature stop codon in SETDB1 was also described in the ACCMESO1 mesothelioma cell line (Cancer Cell Line Encyclopedia (CCLE) database), which suggests a potential role of SETDB1 in MPM development.

Interestingly, most mutations identified in the MPM patient were highly enriched for the mutant allele, suggesting a homozygous alteration or deletion of wildtype allele when minimal contamination of normal pleura in the MPM is considered (Figure 2A). Most variants identified showed a high frequency of mutant alleles, except variants on chromosomes 7 and 20 (Figure 2B). Analyses of allelic fraction between wild-type and variants in MPM tumor (Fig 3A, upper panel), as well as tumor allelic log ratio to normal (Fig 3A, lower panel) using all variants in exome sequencing, including known SNPs, revealed that this MPM showed genome-wide allelic loss or loss of heterozygosity (LOH). However, same 
analyses showed a distinct pattern from another cancer that has many genetic alterations with focal allelic loss (Figure 3B). Although regional loss in chromosomes 1, 3, $4,6,9,10,13,14,17$ and 22 has been reported in MPMs [8-10], to the best of our knowledge, this type of extensive genome-wide allelic loss has not been described in MPM.

Further genetic analysis was not possible because the patient's lung adenocarcinoma specimen was unavailable. Instead, we performed exome sequencing on a focus of atypical adenomatous hyperplasia (AAH), finding no evidence of the genome-wide allelic loss seen in MPM. However, a major driver mutation, G12C, in the KRAS oncogene, might have been involved in the development of this AAH. In our first targeted deep sequencing using the customized Ampliseq panel (Life
Technologies) to validate mutation candidates from MPM, we sequenced six interesting candidates (KANK4, GBP4, SETDB1, ACTB, GOT1, and NOD2) in MPM. Four (SETDB1, ACTB, GOT1, and NOD2) were validated as true somatic mutations (Table 1).

\section{Frequent inactivating mutations of $S E T D B 1$ in MPM}

Among the mutations validated by exome and targeted sequencing in MPM tumor samples, we found that SETDB1 had a nonsense mutation (Y249X). Because another inactivating frameshift mutation (V132fs) was reported in the mesothelioma cell line ACCMESO1, we analyzed mutations of SETDB1 for this whole coding
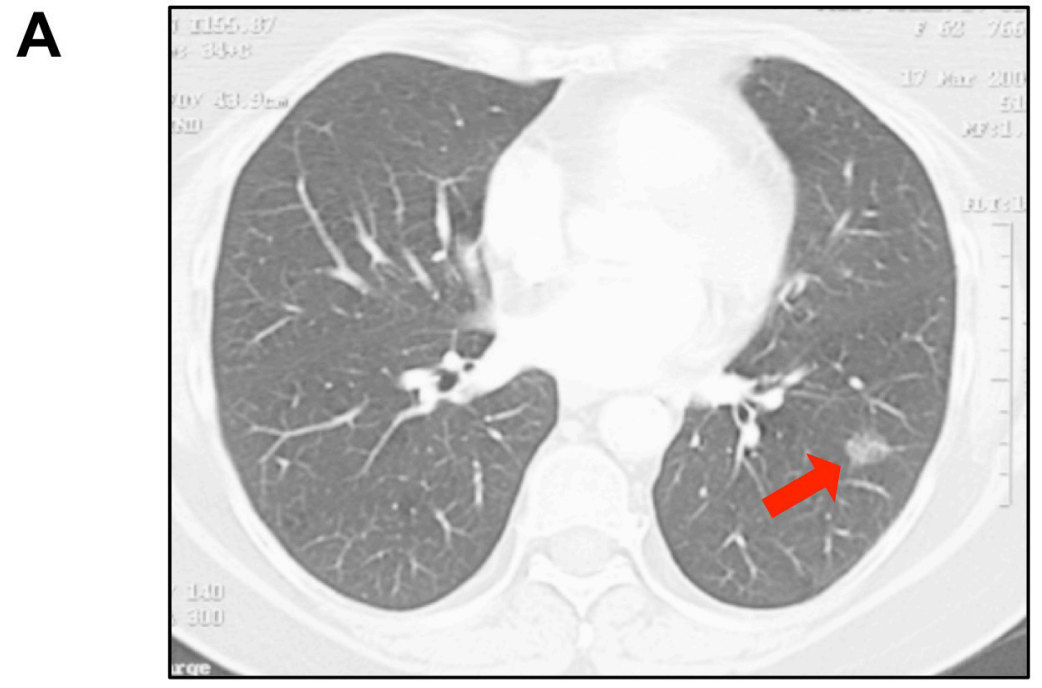

B

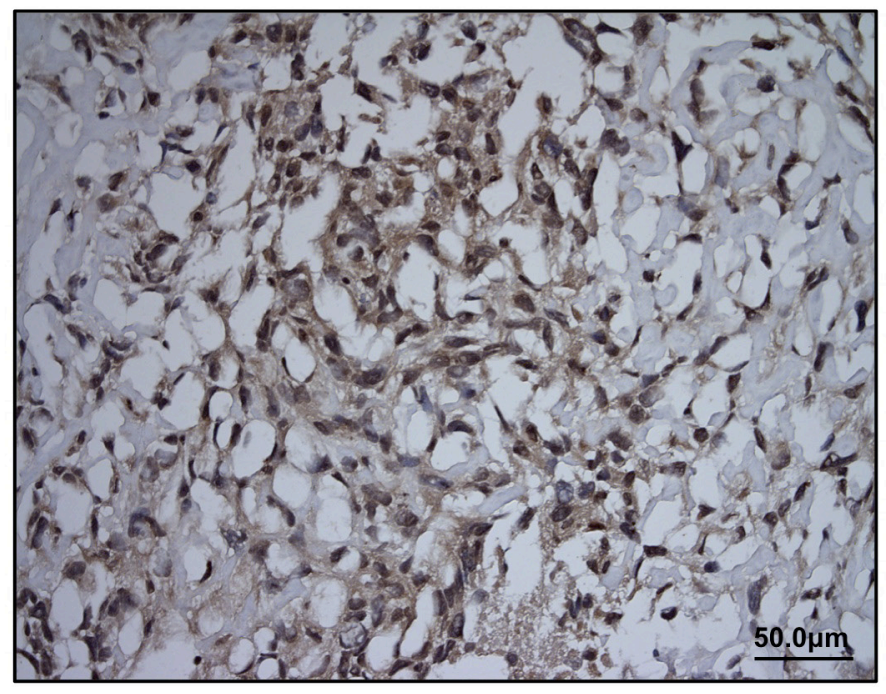

Figure 1: Clinical diagnosis of lung adenocarcinoma and MPM. A. A $2 \mathrm{~cm}$ ground-glass opacity (arrow), later diagnosed as lung adenocarcinoma, was identified in the patient's left lower lobe on chest CT scan. No pleural thickening or implants were noted on preoperative imaging. B. Immunohistochemical staining for calretinin confirmed the diagnosis of MPM (original magnification 20X). 
Table 1: Eleven high-confidence ${ }^{a}$ non-synonymous variants identified by whole exome ${ }^{b}$ and targeted deep sequencing ${ }^{\mathrm{c}}$

\begin{tabular}{|c|c|c|c|c|c|c|c|}
\hline Gene & Chromosome & Protein change & $\begin{array}{c}\text { Nucleotide } \\
\text { change }\end{array}$ & Mutation type & QSS & $\begin{array}{c}\text { Mutant } \\
\text { ratio }\end{array}$ & $\begin{array}{l}\text { Targeted } \\
\text { sequencing }\end{array}$ \\
\hline SETDB1 & $\operatorname{chr} 1$ & Y249X & $747 \mathrm{~T}>\mathrm{A}$ & nonsense & 48 & 0.93 & validated \\
\hline DYSF & $\operatorname{chr} 2$ & R1604Q & $4811 \mathrm{G}>\mathrm{A}$ & missense & 111 & 0.83 & not done \\
\hline ATP2C1 & $\operatorname{chr} 3$ & W460R & $1378 \mathrm{~T}>\mathrm{A}$ & missense & 99 & 0.86 & not done \\
\hline RAPGEF6 & $\operatorname{chr} 5$ & $\mathrm{~T} 325 \mathrm{~S}^{\mathrm{d}}$ & $973 \mathrm{~A}>\mathrm{T}$ & missense & 51 & 0.85 & validated \\
\hline ACTB & $\operatorname{chr} 7$ & F262L & $786 \mathrm{C}>\mathrm{G}$ & missense & 37 & 0.35 & validated \\
\hline CASD1 & $\operatorname{chr} 7$ & V361L & $1081 \mathrm{G}>\mathrm{T}$ & missense & 39 & 0.62 & not done \\
\hline GOT1 & $\operatorname{chr} 10$ & T326I & $977 \mathrm{C}>\mathrm{T}$ & missense & 61 & 0.92 & validated \\
\hline GIT2 & $\operatorname{chr} 12$ & $\mathrm{~T} 28 \mathrm{M}$ & $83 \mathrm{C}>\mathrm{T}$ & missense & 43 & 0.88 & not done \\
\hline NOD2 & $\operatorname{chr} 16$ & Y240X & $720 \mathrm{~T}>\mathrm{A}$ & nonsense & 39 & 0.92 & validated \\
\hline TP53 & $\operatorname{chr} 17$ & $\mathrm{R} 282 \mathrm{~W}^{\mathrm{d}}$ & $844 \mathrm{C}>\mathrm{T}$ & missense & 37 & 0.96 & validated \\
\hline PSG1 & chr19 & E41Q & $121 \mathrm{G}>\mathrm{C}$ & missense & 42 & 0.84 & not done \\
\hline
\end{tabular}

${ }^{a}$ High-confidence is defined by quality score (QSS) greater than 30 .

${ }^{\mathrm{b}}$ Whole exome sequencing was done on SOLiD 5500 (Life Technologies) using the TargetSeq Exome Enrichment kit (Life Technologies) as previously described. [32]

c Targeted deep sequencing for validation was done on Ion Torrent PGM using a customized AmpliSeq panel (Life Technologies) as previously described. [32] (Average coverage $>$ 2000X).

${ }^{\mathrm{d}}$ These mutations were confirmed by Sanger sequencing.

region by targeted deep sequencing of the whole coding exons of SETDB1 in 77 additional primary MPM tissues, for a total sample of 78 MPM tissues from 69 MPM patients. Up to 40,000X sequencing coverage was obtained from our custom-designed targeted sequencing panel. We identified seven somatic mutations from the 69 MPM patients; these mutations have not been reported in any other databases, including 1,000 genome, COSMIC, and dbSNP. Four point mutations and three deletions were identified from a total of six MPM patients (Table 2). Among these, two samples shared the same $17 \mathrm{bp}$ deletion mutation (677_693del17) (Figure 4). All these somatic mutations were validated by three independent targeted sequencings with at least $500 \mathrm{X}$ coverage. Ten matched normal pleural samples were available from 69 mesothelioma patients and sequenced to find any SETDB 1 variant, but none were identified. Finally, to determine whether SETDB1 mutation was correlated with overall survival of MPM patients, we divided 69 MPM patients based on SETDB1 mutation status. We found no significant correlation $(\mathrm{p}=0.351)$, probably because there were so few mutation-positive samples.

\section{DISCUSSION}

Our exome sequencing analysis of MPM in a patient who also had lung adenocarcinoma and a history of bladder cancer showed a rare case of MPM with genome-wide allelic loss. Although karyotyping to confirm genome-wide allelic loss would have been ideal, at the time the experiments were done, the patient had already died and no appropriate samples were available. We therefore used four different sequencing technologies (Exome, two targeted, and Sanger sequencing) and confirmed the genome-wide allelic loss.

Loss of p53 may lead tumor cells to be more vulnerable to unrepaired genomic instability in response to DNA damage or stress [22-24]. Chromothripsis involving massively rearranged chromosomal structure scattered with widespread genomic losses has been observed in several cancer genomes by analyzing their sequence fragments at the whole genome level [26-27]. Chromothripsis is strongly linked to TP53 germline or somatic mutations [26-27]. Although we could not clearly define or classify the genome-wide allelic loss phenomenon as a chromothripsis or similar event because of the limited number of variants identified in exome sequencing analysis, we could assume that bi-allelic inactivation of the TP53 tumor suppressor gene via R282W mutation and LOH played a critical role in the genomic abnormality observed in our MPM case. To the best of our knowledge, TP53 R282W mutation has not been reported in MPM patients. Because TP53 mutation is important for genomic instability such as chromothripsis, and $\mathrm{R} 282 \mathrm{~W}$ is a key mutation altering function of $\mathrm{p} 53$ [2224, 26-27], it would be meaningful to further examine the 
relationship between TP53 R282W and a genome-wide loss in MPM. We believe this is the first report of genomewide allelic loss in a patient who developed multiple primary cancers including MPM. As genome-wide allelic loss can be easily overlooked by mutation-focused genetic analyses, our finding suggests that patients with multiple cancers including MPM may need to be screened for genome-wide allelic loss.

Another notable finding in this study was the high frequency of SETDB1 mutations in MPM. Exome and WGS studies have been done in MPM or peritoneal mesothelioma samples [16-20], but none reported SETDB1 as a new high frequent mutation in mesothelioma.
We identified a somatic SETDB1 mutation by exome sequencing, validated it by targeted deep sequencing analysis, and sequenced 77 additional MPM samples using another targeted deep sequencing panel of SETDB1 three times, which we believe clearly rules out a technical artifact of sequencing. There are several reasons that may explain why other studies did not find that SETDB1 was a frequent mutation in MPM, but we did. First, in previous studies, the $S E T D B 1$ gene was screened by exome or WGS in MPM [16-20]. Some studies used a targeted sequencing panel [28-29] that did not include the SETDB1 gene. Exome or WGS approaches have around 10-100X coverage in general, which may miss a challenging mutant
A

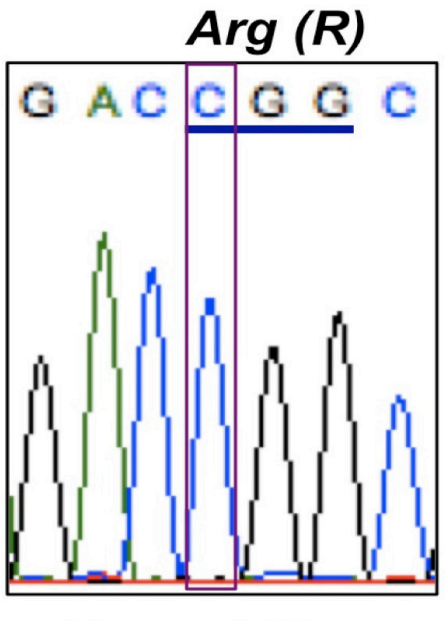

Normal Pleura

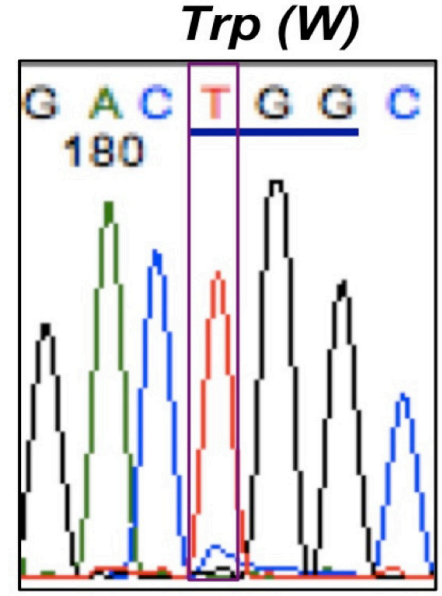

Mesothelioma

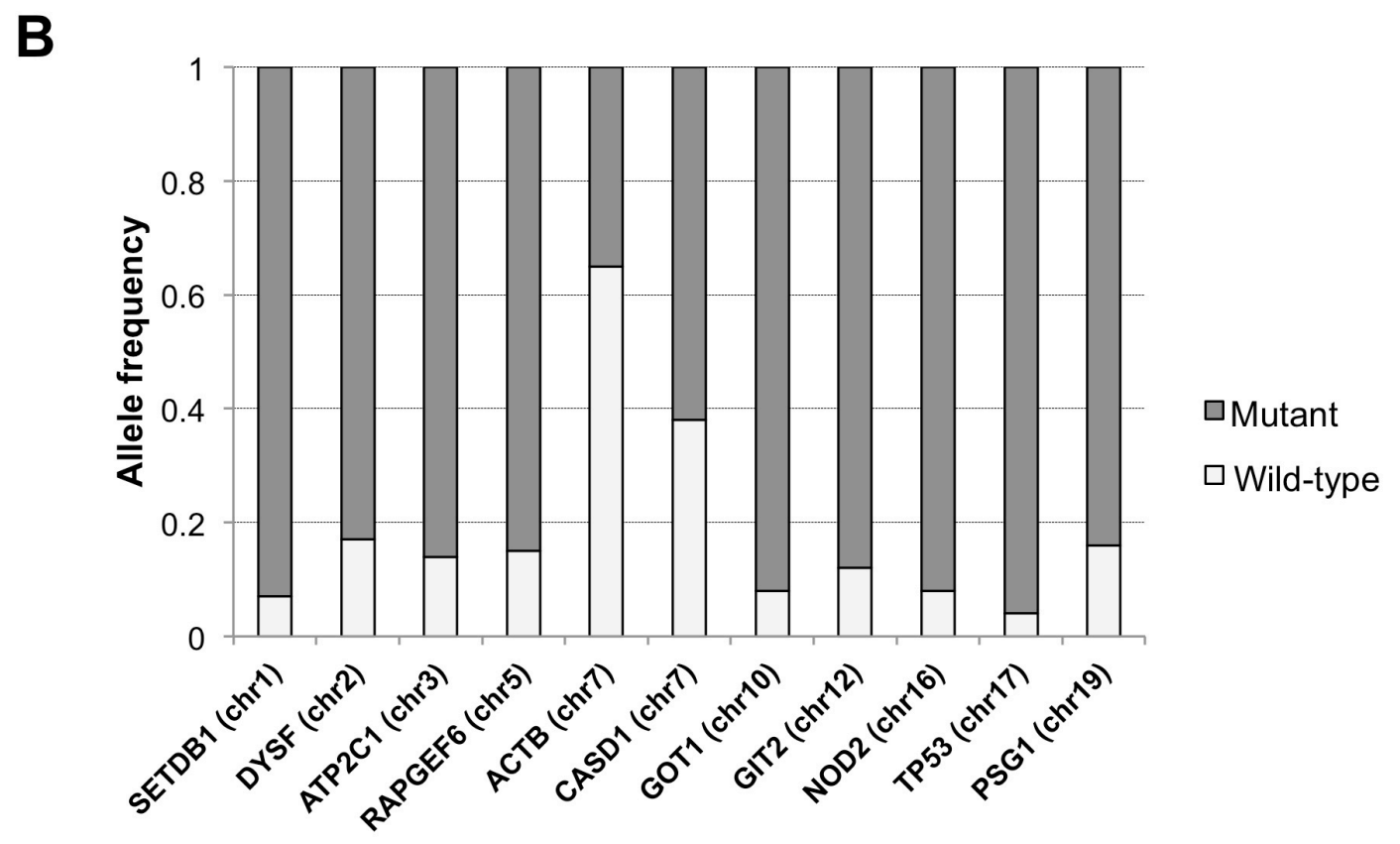

Figure 2: TP53 mutation and allele frequency of all variants. A. Sanger sequencing confirmed TP53 R282W mutation identified in exome and targeted deep sequencing of MPM. B. Allele frequency of variants identified in MPM with genome-wide allelic loss. Frequency of mutant alleles was higher $(>0.8)$ in most variants except for two located on chromosome 7. 
Table 2: SETDB1 mutations identified in 78 MPMs from 69 patients and previously reported

\begin{tabular}{|c|c|c|c|c|c|c|c|}
\hline ID & $\begin{array}{l}\text { Protein } \\
\text { change }\end{array}$ & $\begin{array}{l}{ }^{\text {a }} \text { Nucleotide } \\
\text { change }\end{array}$ & $\begin{array}{l}\text { Mutation } \\
\text { type }\end{array}$ & ${ }^{\mathrm{d}}$ PolyPhen-2 & ${ }^{\mathrm{e} S I F T}$ & $\begin{array}{l}\text { Predicted } \\
\text { function }\end{array}$ & $\begin{array}{c}\text { Mutant } \\
\text { ratio }\end{array}$ \\
\hline $777 \mathrm{~T}$ & Y249X & $747 \mathrm{~T}>\mathrm{A}$ & nonsense & & & Loss of function & 0.91 \\
\hline $869 \mathrm{~T}$ & G869E & $2606 \mathrm{G}>\mathrm{A}$ & missense & damaging & damaging & Damaging & 0.51 \\
\hline $163 \mathrm{~T}$ & C911F & $2732 \mathrm{G}>\mathrm{T}$ & missense & damaging & tolerated & Uncertain & 0.49 \\
\hline $324 \mathrm{~T}$ & S947C & $2840 \mathrm{C}>\mathrm{G}$ & missense & benign & damaging & Uncertain & 0.61 \\
\hline $970 \mathrm{~T}$ & P226RfsX4 & 677_693del17 & frameshift & & & Loss of function & 0.19 \\
\hline $981 \mathrm{~T}$ & P226RfsX4 & 677_693del17 & frameshift & & & Loss of function & 0.23 \\
\hline $1278 \mathrm{~T}$ & F1250del & 3747_3749del & in-frame del & & & Uncertain & 0.14 \\
\hline bACCMESO1 & V132EfsX3 & 395_399del5 & frameshift & & & Loss of function & \\
\hline 'NYU695 & K674SfsX73 & 2020delA & frameshift & & & Loss of function & \\
\hline
\end{tabular}

${ }^{a}$ NM_001145415.1

${ }^{\mathrm{b}}$ Previously reported mutation: ACCMESO1 - V132fs (CCLE, http://www.broadinstitute.org/ccle)

c Previously reported mutation: NYU695 - p.K674fs [18]

${ }^{\mathrm{d}}$ http://genetics.bwh.harvard.edu/pph2/

${ }^{\mathrm{e}} \mathrm{http}: / /$ sift.jcvi.org/

A
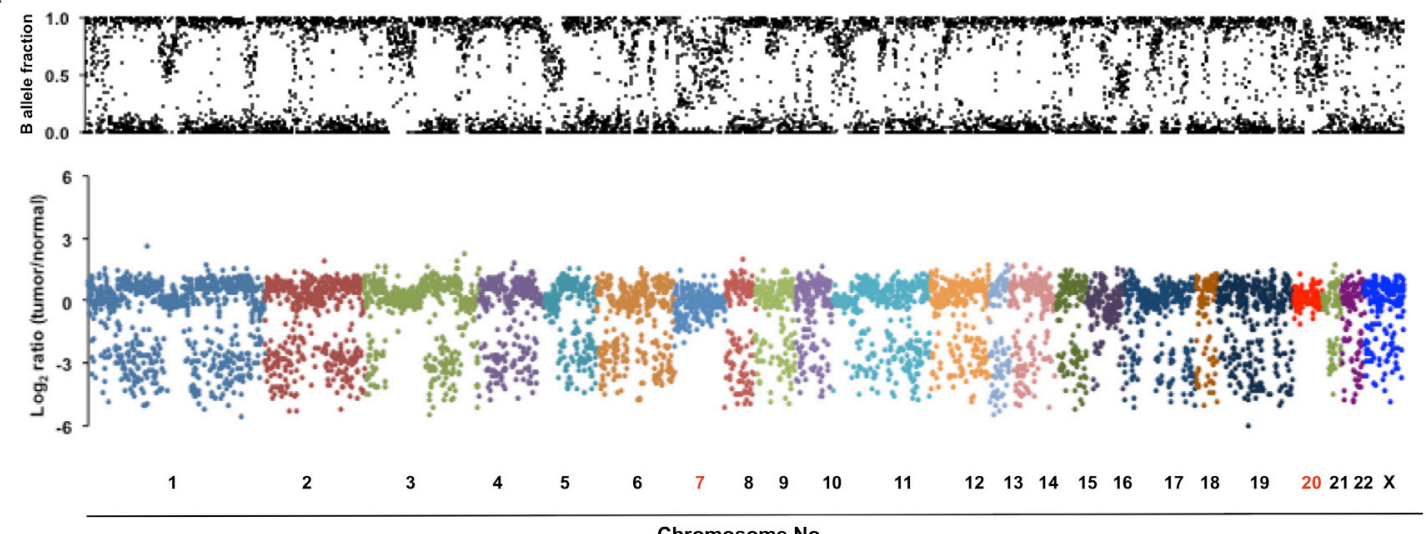

B
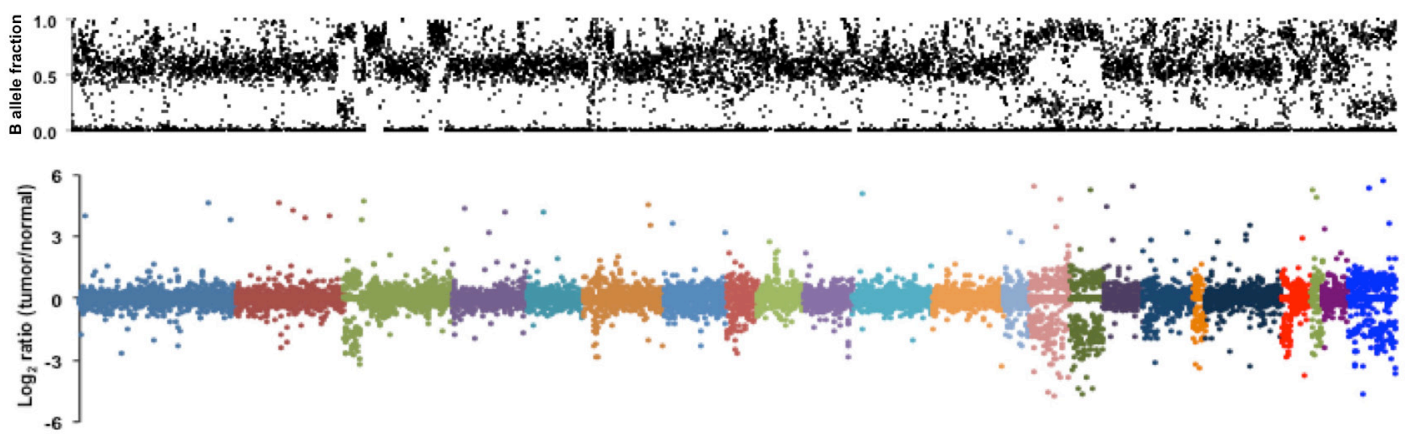

Figure 3: Genome-wide allelic loss identified in a MPM patient with multiple primary cancers. A. Scatter plots show genome-wide allelic view of all variants including known SNPs identified in the patient's MPM. Allelic fraction represents ratio of wildtype to variant counts in an exome sequencing. B-allele fraction plot in the upper panel represents allelic ratio between wild-type and variant in the tumor sample. In the lower panel, the values ( $\log 2$ ratio) of the $\mathrm{x}$-axis are an allelic fraction of tumor normalized to that of matched normal pleura. The majority of variants on all chromosomes except chromosomes 7 and 20 show losses of either wild-type or variant allele in tumor. B. An example of genome-wide allelic view of tumor with frequent genetic alterations, but only with focal allelic loss. B-allele fraction plot of tumor sample in the upper panel and allelic fraction plot of tumor normalized to that of matched normal in the lower panel were newly generated from exome sequencing data previously reported [32]. 
allele. In contrast, our coverage range of SETDB1 targeted deep sequencing was up to $40,000 \mathrm{X}$ - a difference that could explain why we detected the SETDB1 mutation. Second, previous studies analyzed single-digit sample sizes of MPM [16-20], except for one exome analysis [18] that included 22 MPM samples. Third, different types of samples were used for mutation screening, which may have affected the detection results. We used flashfrozen tissues from MPM patients, whereas some previous studies [16-17] used cultured cells. Finally, we screened MPM samples, whereas some previous exome or WGS studies focused on peritoneal mesothelioma, another subtype $[17,20]$.

SETDB1 has been reported to be amplified and a potential oncogene in lung cancer [21]. Increased expression of SETDB1 promotes tumor invasiveness and sensitizes anti-tumor growth by mithramycin, a SETDB1 and Sp1 inhibitor [21]. Our finding that SETDB1 had a nonsense mutation (Y249X) and the fact that another inactivating frameshift mutation (V132fs) was reported in the mesothelioma cell line ACCMESO1, led us to hypothesize that truncating mutations such as nonsense and frameshift mutations are frequent in MPM. When we tested this using targeted deep sequencing analysis in 78 MPM samples from 69 MPM patients, we found three deletion mutations. Two were the same $17 \mathrm{bp}$ deletion mutations at exon 7 and the other was an in-frame deletion mutation (Table 2) (Figure 4). The remaining three mutations were novel missense mutations. Interestingly, while all predicted loss-of-function mutations except one are located in the N-terminal, missense and in-frame deletion mutations affecting single amino acid residues are located in the SET domain of SETDB1 (Figure 5) [30-31]. Although SETDB1 has been suggested to have an oncogenic impact in lung cancer based on transcription level or expression data [21,25], our results suggest that at
A

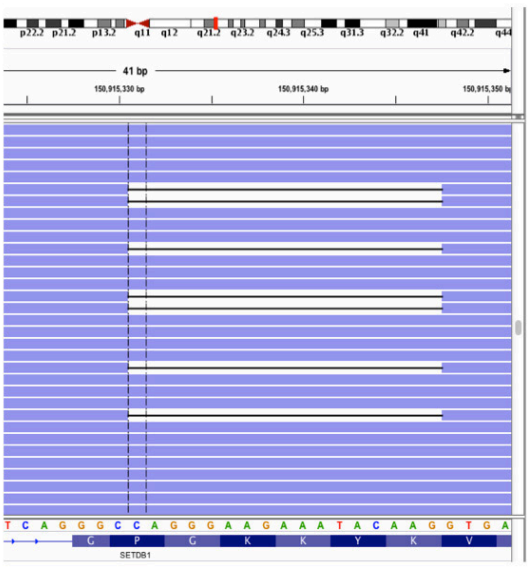

B

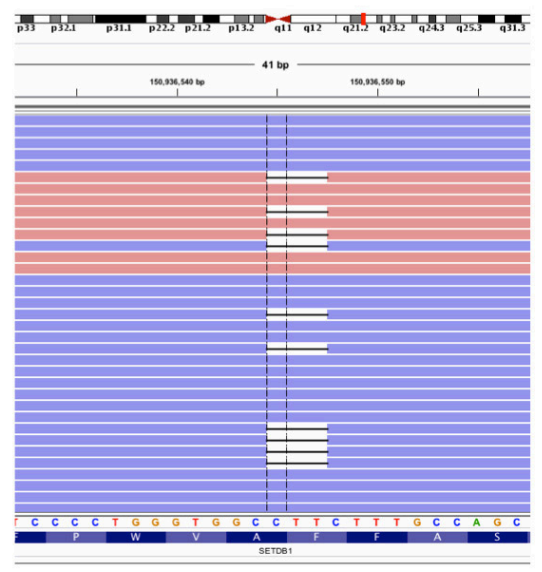

Figure 4: Frequent SETDB1 mutations in MPM patients. Two types of deletion mutations from MPM patients, shown by an Integrative Genomics Viewer (IGV). A. 17 bp deletion (677_693del17) and B. In-frame deletion (3747_3749del) of SETDB1 were identified from different MPM patients.

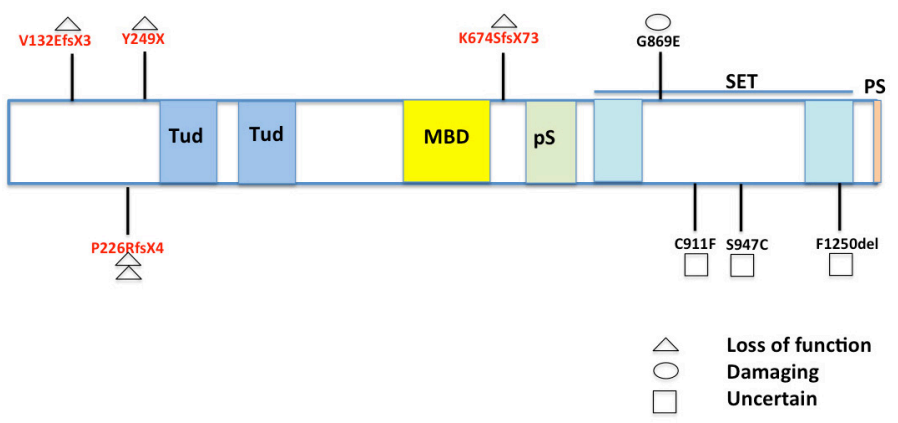

Figure 5: Map of SETDB1 mutations identified in MPMs. Nine SETDB1 somatic mutations identified in this study and reported previously were mapped in domains of SETDB1. Four of five truncating mutations were located at the N-terminal (5') regions before Tudor (Tud) domains. All three missense and one deletion mutation were found in the SET domain. Tud: Tudor; MBD: methyl-CpG-binding domain; pS: pre-SET; PS: post-SET [30-31]. 
least some of the identified SETDB1 somatic mutations are probably loss of function mutations. Functional validation and characterization should be performed to prove this, but the different mutation spectrum suggests the location of somatic mutations on the SETDB1 functional domains may be important for oncogenic effect or tumor suppression. Exome sequencing in eight primary cultured cells from different subtypes of mesothelioma found that genes involved in histone modification and regulation mechanisms, such as BAP1, SETD2, USP49, and PRMT6, were mutated, suggesting somatic inactivation of histone modifier genes is important for mesothelioma development [16]. The same study also identified a truncating mutation of BAP1 and whole deletion of PRMT6 [16]. These results are consistent with our finding that mutations of $S E T D B 1$, histone methyltransferase, are frequent in MPM, suggesting that histone modifier genes, including SETDB1, are potentially important in MPM. Further functional studies are required to investigate a role of $S E T D B 1$ in MPM development.

In summary, we identified genome-wide allelic loss in a patient who had MPM and two additional primary cancers, results which suggest that careful analysis in exome sequencing is needed to detect genome-wide deletion in MPM samples with or without multiple primary cancers. The high frequency of mutations in SETDB1 that we found suggests that this and other histone-related genes are important in MPM.

\section{MATERIALS AND METHODS}

\section{DNA extraction of 78 primary MPM tissues from 69 MPM patients}

DNA from a patient with multiple primary cancers (bladder, lung, and MPM), and 77 additional MPM tissues was extracted as described previously [32-33]. All samples were collected under a protocol (\#11-06107) approved by the Committee for Human Research at the University of California, San Francisco. Clinical data for the 69 MPM patients is summarized in Supplementary Table 1.

\section{Immunohistochemistry (IHC) staining}

The MPM tissue slides from the patient with multiple primary cancers were used for IHC staining with anti-calretinin antibody (\# 180211, ThermoFisher) as previously described [34]. Briefly, $5 \mu \mathrm{m}$-thick slide sections were deparaffinized with xylene and steamed in citrate for 20 minutes. They were then treated with blocking solution, washed, and incubated overnight. The slides were washed again and incubated with solutions from the Invitrogen Histostain Plus Broad Spectrum Kit (85-9643), washed again, stained with hematoxylin for 1 minute, mounted, and analyzed.

\section{DNA library preparation and exome sequencing}

Serial samples of normal tissue, atypical adenomatous hyperplasia (AAH), and MPM tissue from the patient with multiple cancers including MPM were used for the exome sequencing library preparation. SOLiD 5500 (Life Technologies) was used for whole exome sequencing according to the manufacturer's protocol and our previous experiment [32]. Briefly, 3 ug of DNA was fragmented by Covaris S220 (Covaris), and the fragmented DNA was barcode ligated and amplified. After fragmented DNA was quantified, $500 \mathrm{ng}$ of DNA was used for the TargetSeq Exome Enrichment kit (Life Technologies) to enrich the exome only. A diluted exome library was amplified by emulsion PCR and enriched. The prepared libraries were run onto the Flow Chip in SOLiD 5500 .

\section{Targeted deep sequencing to validate somatic mutation candidates}

To validate the identified mutations from the exome sequencing of the patient who had MPM and two other primary cancers, we designed a customized Ampliseq panel (Life Technologies) to sequence six mutation candidates (KANK4, GBP4, SETDB1, ACTB, GOT1, and $N O D 2)$. The Ion Ampliseq designer was used to make a targeted customized panel for these six genes according to the manufacturer's protocol. Library preparation and sequencing were also done according to our previous protocol [32].

\section{Targeted deep sequencing of SETDB1 in 78 MPM tissues from 69 MPM patients}

We designed another targeted panel of SETDB1 covering all 21 coding exons spanning $6.05 \mathrm{~kb}(99.76 \%$ coverage) (CureSeq Inc, Brisbane, CA). A total of 31 amplicons were designed and sequenced in 78 primary mesothelioma tissue samples. A library was prepared according to the manufacturer's protocol (CureSeq Inc, Brisbane, CA). In brief, library preparation was performed on each sample with adaptor and barcode ligation. Sequencing adaptors and barcodes (CureSeq Inc, Brisbane, CA) for multiplex sequencing purposes were ligated to the amplicons using ligase. After purification, the library was quantified using the Agilent Bioanalyzer with High-Sensitivity DNA kits (Agilent Technologies) following the manufacturer's protocol. Library emulsion and enrichment were performed using the Ion PGM One Touch 2 and Enrichment System machine according to the manufacturer's protocol (Life Technologies). Library with Ion Sphere Particles (ISPs) was added to the reaction tube using Ion PGM Template reagents (Life Technologies). ISP emulsion and breaking were automated through the Ion PGM OneTouch 2 system. The recovered ISPs were 
enriched by an Ion PGM ES machine using Dynabeads MyOne Streptavidin C1 beads (Life Technologies). Sequencing was done according to the manufacturer's protocol with enriched ISPs using the 318 chips (Life Technologies) on an Ion Torrent PGM.

\section{Analysis of exome and targeted sequencing data}

Raw sequence data were converted to fastq format using BEDtools (version 2.17.0) to perform alignment to the human reference genome (hg19, NCBI Build 37) using the Burrows_Wheeler Aligner (BWA mem, version 0.7.12). Aligned data were then grouped by sample data using picard (version 1.114). The Genome Analysis ToolKit (GATK version 3.3-0) was used to perform insertion and deletion realignment, base recalibration, and variant calling. Single nucleotide variants and insertions/deletions were detected by using GATK, and annotated afterwards with ANNOVAR [35]. Several databases were used for annotation filtering such as dbSNP and 1000 Genomes (www.1000genomes.org). All the annotated mutations were visualized by Integrative Genomics Viewer (IGV, version 2.3.43) to confirm. Python-based programs developed in-house were used to filter out common benign and recurrent artifact variants during the annotation validations with IGV. Mutation frequencies were calculated through the programs by using information obtained from processed bam files by comparing the reads containing the mutations and the total number of reads containing the region of mutations. Those samples which were validated for variants were reconfirmed through separate run process to rule out the experimental bias.

\section{Sanger sequencing validation}

Sanger sequencing as described previously [33] was done to validate TP53 R282W and RAPGEF6 T325S mutations in the sample from the MPM patient who had additional primary cancers.

\section{Survival analysis of MPM patients according to SETDB1 mutation status}

We divided 69 MPM patients into SETDB1 wildtype $(n=62)$ and mutant $(n=7)$ groups. Overall survival rates were determined using the Kaplan-Meier method in SPSS V22.0 for Mac. Survival rates were compared using the log-rank test. Two-sided P values less than 0.05 were considered statistically significant.

\section{ACKNOWLEDGMENTS}

This study was supported by Mesothelioma Applied Research Foundation, the Kazan, McClain, Abrams, Fernandez, Lyons, Greenwood, Harley \& Oberman
Foundation; the Honeywell Foundation; and the Barbara Isackson Lung Cancer Research Fund. We thank Pamela Derish in the UCSF Department of Surgery for editorial assistance with the manuscript.

\section{CONFLICTS OF INTEREST}

DMJ and IJK are equity holders and consultants of CureSeq Inc.

\section{REFERENCES}

1. Raja S, Murthy SC, Mason DP. Malignant pleural mesothelioma. Current Oncology Reports. 2011; 13:259-264.

2. Campbell NP, Kindler HL. Update on malignant pleural mesothelioma. Seminars in Respiratory and Critical Care Medicine. 2011; 32:102-110.

3. Carbone M, Yang H. Molecular pathways: targeting mechanisms of asbestos and erionite carcinogenesis in mesothelioma. Clinical Cancer Research. 2012; 18:598-604.

4. Tsujimura T, Torii I, Sato A, Song M, Fukuoka K, Hasegawa S, Nakano T. Pathological and molecular biological approaches to early mesothelioma. International Journal of Clinical Oncology. 2012; 17:40-47.

5. Takigawa N, Kiura K, Kishimoto T. Medical treatment of mesothelioma: anything new? Current Oncology Reports. 2011; 13:265-271.

6. Vogelzang NJ, Rusthoven JJ, Symanowski J, Denham C, Kaukel E, Ruffie P, Gatzemeier U, Boyer M, Emri S, Manegold C, Niyikiza C, Paoletti P. Phase III study of pemetrexed in combination with cisplatin versus cisplatin alone in patients with malignant pleural mesothelioma. Journal of Clinical Oncology. 2003; 21:2636-2644.

7. van Meerbeeck JP, Gaafar R, Manegold C, Van Klaveren RJ, Van Marck EA, Vincent M, Legrand C, Bottomley A, Debruyne C, Giaccone G; European Organisation for Research and Treatment of Cancer Lung Cancer Group; National Cancer Institute of Canada. Randomized phase III study of cisplatin with or without raltitrexed in patients with malignant pleural mesothelioma: an intergroup study of the European Organisation for Research and Treatment of Cancer Lung Cancer Group and the National Cancer Institute of Canada. Journal of Clinical Oncology. 2005; 23:6881-6889.

8. Flejter WL, Li FP, Antman KH, Testa JR. Recurring loss involving chromosomes 1,3 , and 22 in malignant mesothelioma: possible sites of tumor suppressor genes. Genes, Chromosomes and Cancer. 1989; 1:148-154.

9. Jean D, Daubriac J, Le Pimpec-Barthes F, Galateau-Salle F, Jaurand MC. Molecular changes in mesothelioma with an impact on prognosis and treatment. Archives of Pathology \& Laboratory Medicine. 2012; 136:277-293..

10. Björkqvist AM, Tammilehto L, Anttila S, Mattson K, Knuutila S. Recurrent DNA copy number changes in 1q, 4q, 
$6 \mathrm{q}, 9 \mathrm{p}, 13 \mathrm{q}, 14 \mathrm{q}$ and $22 \mathrm{q}$ detected by comparative genomic hybridization in malignant mesothelioma. British Journal of Cancer. 1997; 75:523-527.

11. Cheng JQ, Jhanwar SC, Klein WM, Bell DW, Lee WC, Altomare DA, Nobori T, Olopade OI, Buckler AJ, Testa JR. p16 alterations and deletion mapping of 9p21-p22 in malignant mesothelioma. Cancer Research. 1994; 54:5547-5551.

12. Sekido Y, Pass HI, Bader S, Mew DJ, Christman MF, Gazdar AF, Minna JD. Neurofibromatosis type 2 (NF2) gene is somatically mutated in mesothelioma but not in lung cancer. Cancer Research. 1995; 55:1227-1231.

13. Bott M, Brevet M, Taylor BS, Shimizu S, Ito T, Wang L, Creaney J, Lake RA, Zakowski MF, Reva B, Sander C, Delsite R, Powell S, et al. The nuclear deubiquitinase BAP1 is commonly inactivated by somatic mutations and $3 \mathrm{p} 21.1$ losses in malignant pleural mesothelioma. Nature Genetics. 2011; 43:668-672.

14. Testa JR, Cheung M, Pei J, Below JE, Tan Y, Sementino E, Cox NJ, Dogan AU, Pass HI, Trusa S, Hesdorffer M, Nasu M, Powers A, et al. Germline BAP1 mutations predispose to malignant mesothelioma. Nature Genetics. 2011; 43:1022-1025.

15. Sugarbaker DJ, Richards WG, Gordon GJ, Dong L, De Rienzo A, Maulik G, Glickman JN, Chirieac LR, Hartman ML, Taillon BE, Du L, Bouffard P, Kingsmore SF, et al. Transcriptome sequencing of malignant pleural mesothelioma tumors. Proceedings of the National Academy of Sciences of the United States of America. 2008; 105:3521-3526.

16. Yoshikawa Y, Sato A, Tsujimura T, Otsuki T, Fukuoka K, Hasegawa S, Nakano T, Hashimoto-Tamaoki T. Biallelic germline and somatic mutations in malignant mesothelioma: multiple mutations in transcription regulators including $\mathrm{mSWI} / \mathrm{SNF}$ genes. International Journal of Cancer. 2015; 136:560-571.

17. Yu W, Chan-On W, Teo M, Ong CK, Cutcutache I, Allen GE, Wong B, Myint SS, Lim KH, Voorhoeve PM, Rozen $\mathrm{S}$, Soo KC, Tan P, Teh BT. First somatic mutation of E2F1 in a critical DNA binding residue discovered in well-differentiated papillary mesothelioma of the peritoneum. Genome Biology. 2011; 12:R96.

18. Guo G, Chmielecki J, Goparaju C, Heguy A, Dolgalev I, Carbone M, Seepo S, Meyerson M, Pass HI. Whole-exome sequencing reveals frequent genetic alterations in BAP1, NF2, CDKN2A, and CUL1 in malignant pleural mesothelioma. Cancer Research. 2015; 75:264-269.

19. Bueno R, De Rienzo A, Dong L, Gordon GJ, Hercus CF, Richards WG, Jensen RV, Anwar A, Maulik G, Chirieac LR, Ho KF, Taillon BE, Turcotte CL, Hercus RG, Gullans $\mathrm{SR}$, Sugarbaker DJ. Second generation sequencing of the mesothelioma tumor genome. PLoS One. 2010; 5:e10612.

20. Sheffield BS, Tinker AV, Shen Y, Hwang H, Li-Chang HH, Pleasance E, Ch'ng C, Lum A, Lorette J, McConnell YJ, Sun S, Jones SJ, Gown AM, et al. Personalized oncogenomics: clinical experience with malignant peritoneal mesothelioma using whole genome sequencing. PLoS One. 2015; 10:e119689.

21. Rodriguez-Paredes M, Martinez de Paz A, Simó-Riudalbas L, Sayols S, Moutinho C, Moran S, Villanueva A, VázquezCedeira M, Lazo PA, Carneiro F, Moura CS, Vieira J, Teixeira MR, Esteller M. Gene amplification of the histone methyltransferase SETDB1 contributes to human lung tumorigenesis. Oncogene. 2014; 33:2807-2813.

22. Calhoun S, Daggett V. Structural effects of the L145Q, V157F, and R282W cancer-associated mutations in the p53 DNA-binding core domain. Biochemistry 2011; 50: 5345-5353.

23. Joerger AC, Ang HC, Fersht AR. Structural basis for understanding oncogenic p53 mutations and designing rescue drugs. Proceedings of the National Academy of Sciences of the United States of America. 2006; 103:15056-15061.

24. Shao C, Deng L, Henegariu O, Liang L, Stambrook PJ, Tischfield JA. Chromosome instability contributes to loss of heterozygosity in mice lacking p53. Proceedings of the National Academy of Sciences of the United States of America. 2000; 97:7405-7410.

25. Ceol CJ, Houvras Y, Jane-Valbuena J, Bilodeau S, Orlando DA, Battisti V, Fritsch L, Lin WM, Hollmann TJ, Ferré F, Bourque C, Burke CJ, Turner L, et al. The histone methyltransferase SETDB1 is recurrently amplified in melanoma and accelerates its onset. Nature. 2011; 471:513-517.

26. Morrison CD, Liu P, Woloszynska-Read A, Zhang J, Luo W, Qin M, Bshara W, Conroy JM, Sabatini L, Vedell P, Xiong D, Liu S, Wang J, et al. Whole-genome sequencing identifies genomic heterogeneity at a nucleotide and chromosomal level in bladder cancer. Proceedings of the National Academy of Sciences of the United States of America. 2014; 111:E672-681.

27. Rausch T, Jones DT, Zapatka M, Stütz AM, Zichner T, Weischenfeldt J, Jäger N, Remke M, Shih D, Northcott PA, Pfaff E, Tica J, Wang Q, et al. Genome sequencing of pediatric medulloblastoma links catastrophic DNA rearrangements with TP53 mutations. Cell. 2012; 148:59-71.

28. Miyanaga A, Masuda M, Tsuta K, Kawasaki K, Nakamura Y, Sakuma T, Asamura H, Gemma A, Yamada T. Hippo pathway gene mutations in malignant mesothelioma: revealed by RNA and targeted exon sequencing. Journal of Thoracic Oncology. 2015; 10: 844-851.

29. Lo Iacono M, Monica V, Righi L, Grosso F, Libener R, Vatrano S, Bironzo P, Novello S, Musmeci L, Volante M, Papotti M, Scagliotti GV. Targeted next-generation sequencing of cancer genes in advanced stage malignant pleural mesothelioma: a retrospective study. Journal of Thoracic Oncology. 2015; 10:492-499.

30. Tachibana K, Gotoh E, Kawamata N, Ishimoto K, Uchihara Y, Iwanari H, Sugiyama A, Kawamura T, Mochizuki Y, Tanaka T, Sakai J, Hamakubo T, Kodama T, Doi T. Analysis of the subcellular localization of the human histone methyltransferase SETDB1. Biochemical and Biophysical Research Communications. 2015; 465:725-731. 
31. Kang YK. SETDB1 in Early Embryos and Embryonic Stem Cells. Current Issues in Molecular Biology. 2015; 17:1-10.

32. Fang LT, Lee S, Choi H, Kim HK, Jew G, Kang HC, Chen L, Jablons D, Kim IJ. Comprehensive genomic analyses of a metastatic colon cancer to the lung by whole exome sequencing and gene expression analysis. International Journal of Oncology. 2014; 44:211-221.

33. Choi H, Kratz J, Pham P, Lee S, Ray R, Kwon YW, Mao JH, Kang HC, Jablons D, Kim IJ. Development of a rapid and practical mutation screening assay for human lung adenocarcinoma. International Journal of Oncology. 2012; 40:1900-1906.

34. Kim IJ, Quigley D, To MD, Pham P, Lin K, Jo B, Jen KY, Raz D, Kim J, Mao JH, Jablons D, Balmain A. Rewiring of human lung cell lineage and mitotic networks in lung adenocarcinomas. Nature Communications. 2013; 4:1701.

35. Wang K, Li M, Hakonarson H. ANNOVAR: functional annotation of genetic variants from high-throughput sequencing data. Nucleic Acids Research. 2010; 38:e164. 